Preprint

UCRL-JC-136856

\title{
Detection of Linear Features Using a Localized Radon Transform with a Wavelet Filter
}

\author{
A.L. Warrick, P.A. Delaney
}

This article was submitted to

Thirtieth Asilomar Conference on Signals, Systems, Pacific Grove, CA, November 3-6, 1996

\section{December 13, 1999}

U.S. Department of Energy

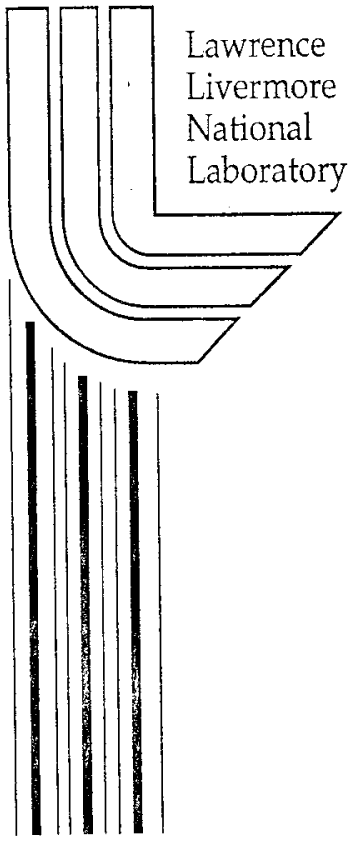




\title{
DETECTION OF LINEAR FEATURES USING A LOCALIZED RADON TRANSFORM WITH A WAVELET FILTER
}

\author{
Abbie L. Warrick ${ }^{1} \quad$ Pamela A. Delaney ${ }^{2}$ \\ 'Imaging and Detection Program \\ Lawrence Livermore National Laboratory, Livermore, California, 94550 \\ ${ }^{2}$ Electrical Engineering Department \\ Wichita State University, Wichita, Kansas, 67260-0044
}

\begin{abstract}
ABSTR.ACT
One problem of interest to the oceanic engineering community is the detection and enhancement of internal wakes in open water synthetic aperture radar (SAR) images. Internal wakes, which occur when a ship travels in a stratified medium, have a "V" shape extending from the ship, and a chirp-like feature across each arm. The Radon transform has been applied to the detection and the enhancement problems in internal wake images to account for the linear features while the wavelet iransform has been applied to the enhancement problem in internal wake images to account for the chirp-like features. In this paper, a new transform, a localized Radon transform with a wavelet filter (LRTWF), is developed which accounts for both the linear and the chirp-like features of the internal wake. This transform is then incorporated into oprimal and sub-optimal detection schemes for images (with these features) which are contaminated by additive Gaussian noise.
\end{abstract}

\section{INTRODUCTION}

The Radon transform (RT) has become extremely useful in many areas, particularly in tomography $[1,2]$. The Radon transform accentuates linear features in images and has been applied successfully to the enhancement and detection problem in SAR open water images $[3,4,5]$. However, current methods involving Radon transform techniques do not account for the chirp-like features in the wake.

Recently, the wavelet transform has been applied to detection and enhancement problems in SAR images $[6,7,8]$, including internal wakes in open water images [9]. Although the wavelet transform was useful in examining SAR wake images, it required extracting azimuth slices of the wake. Ideally, a method which does not require explicit extraction of line segments is desired.

In this paper, a new transform, the LRTWF, is developed which combines the wavelet transform and the Radou transform to account for the chirp-like feature and the linear feature in internal wake inages respectively. For the LRTWF, extraction of line segments external to the transform is not required.

\section{THE LOCALIZED RADON TRANSFORM WITH A WAVELET FILTER}

In order to account for the chirp-like feature of the internal rake, the kernel of the LRTWF is obtained by multiplying a wavelet function by a window function

$$
\begin{gathered}
\psi^{a, b, q, \theta}(x, y)=\frac{1}{\sqrt{|a|}} \dot{w}\left(\frac{x \cos \theta+y \sin \theta-b}{a}\right) \\
\times W_{\lambda}(-x \sin \theta \div y \cos \theta-q)
\end{gathered}
$$

where $\psi \in \mathcal{L}^{2}(R)$ is considered to be an admissible wavelet which satisfies [10]

$$
\int_{-\infty}^{\infty} \frac{d \xi}{|\xi|}|\hat{\psi}(\xi)|^{2}<\infty
$$

and

$$
W_{\lambda}(x)= \begin{cases}1, & |x|<\lambda / 2 \\ 0, & \text { elsewhere. }\end{cases}
$$

The forward transform is defined as the inner product of the function $f$ with the kerne!, $\psi^{a, b, q, 9}$. Thus,

$$
\begin{aligned}
& \left(T^{\text {irtwf }} f\right)(a, b, q, \theta)=\left\langle f, \dot{2}^{a, b \cdot a, \theta}\right\rangle \\
& =\iint d x d y f(x, y) \frac{1}{\sqrt{|a|}} \overline{\left(\frac{x \cos \theta+y \sin \theta-b}{a}\right)} \\
& \times W_{\lambda}(-x \sin \theta+y \cos \theta-q) \text {. }
\end{aligned}
$$

For the purposes of this work, a rectangular window is sufficient. However, it is possible to implement a more generalized window.

The LRTWF was developed using the RT coordinates $(b, \theta)$. However, the transform can be defined equivalently in terms of rectangular coordinates. Define the parameters $(\alpha, \beta)$ as

$$
\alpha=q \sin \theta+b \cos \theta \quad \beta=q \cos \theta-b \sin \theta .
$$

The LRTWF can be written in terms of the rectangular coordinates $(\alpha, \beta)$ as

$$
\begin{aligned}
\left(T^{l \tau t w f} f\right)(a, \alpha, \beta, \theta)=\iint d x d y f(x, y) \\
\times \\
\times \psi\left(\frac{(x-\alpha) \cos \theta+(y-\beta) \sin \theta}{a}\right) \\
\times W_{\lambda}(-(x-\alpha) \sin \theta+(y-\beta) \cos \theta) .
\end{aligned}
$$

Note that the transforms given in Equations (4) and (6) are equivalent and related by the rotational transformation given in Equation (5). Another formulation of the LRTWF 


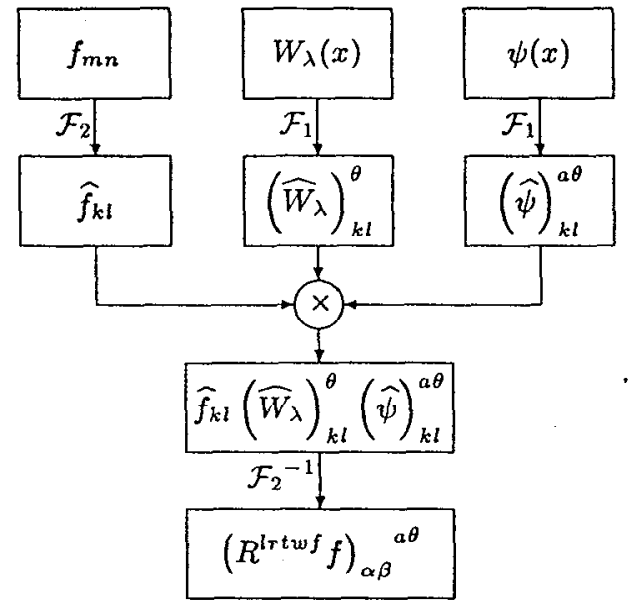

Figure 1. Diagram illustrating implementation of the LRTWF.

can be obtained in terms of the Fourier transforms. By applying Parseval's theorem to Equation (6), we find

$$
\begin{aligned}
\left(T^{l r t w f} f\right) & (a, \alpha, \beta, \theta)=\iint d \xi d \nu \widehat{f}(\xi, \nu) \\
\times & \sqrt{|a|} \frac{\widehat{\psi}(a(\xi \cos \theta+\nu \sin \theta))}{\lambda} \\
\times & \frac{4}{\lambda} \frac{\sin \left(\frac{\lambda}{2}(-\xi \sin \theta+\nu \cos \theta)\right)}{\frac{\lambda}{2}(-\xi \sin \theta+\nu \cos \theta)} e^{i(\alpha \xi+\beta \nu)}
\end{aligned}
$$

The rectangular formulation in Equation (7) provides a convenient representation for numerical implementation of the LRTWF which is similar to the direct Fourier method in the RT. The LRTWF can be obtained by taking the discrete Fourier transform of the image, multiplying by the wavelet function

$$
(\widehat{\psi})_{k l}^{a, \theta}=\sqrt{|a|} \overline{\widehat{\psi}\left(a\left(\xi_{k} \cos \theta+\nu_{l} \sin \theta\right)\right)}
$$

and the window function

$$
\left(\widehat{W}_{\lambda}\right)_{k l}^{\theta}=\frac{4}{\lambda} \frac{\sin \left(\frac{\lambda}{2}\left(-\xi_{k} \sin \theta+\nu_{l} \cos \theta\right)\right)}{\frac{\lambda}{2}\left(-\xi_{k} \sin \theta+\nu_{l} \cos \theta\right)}
$$

and then taking the inverse Fourier transform which is illustrated in Figure 1. If the analytical form of the Fourier transform of the wavelet and window function are known, then the rotation information does not require interpolation.

\section{COMPOSITE DETECTION}

The LRTWF was developed to simultaneously accentuate the linear and chirp-like features in internal wake images. Next, a quantitatively method of detecting these features in images is examined. It is then shown that the LRTWF can be used to simplify the detection problem.

In a traditional binary hypothesis problem $[11,12]$, we assume that

$$
\begin{array}{ll}
\mathrm{H}: & \mathrm{X}=\mathrm{N} \\
\mathrm{K}: & \mathrm{X}=\mathrm{N}+\mathrm{s}(\alpha),
\end{array}
$$

where $\mathrm{X}$ is the observation vector of length $M, \mathrm{~N}$ is a noise vector of length $M$, and $\mathrm{s}$ is a signal of length $M$ parameterized by the random variable $\alpha$. In order to simplify the problem, we assume that the elements in the vector $N$ are observations from an i.i.d. Gaussian random process with mean $\mu=0$ and variance $\sigma^{2}$. In addition, we assume that $\mathbf{s}(\alpha)^{T} \mathbf{s}(\alpha)=1$ and that the parameter $\alpha$ is uniformly distributed on

$$
\Omega_{\alpha}=\left\{\alpha \mid \alpha_{0}, \alpha_{1}, \ldots, \alpha_{Q-1}\right\}
$$

Note that the signal $s(\alpha)$ and the unknown parameter $\alpha$ are general and can be specified by the application. For the binary hypothesis problem given in Equation (8), the conditional likelihood ratio given the parameter $\alpha$ is

$$
L(\mathbf{x} \mid \alpha)=e^{-\frac{1}{2} \operatorname{SNR}} e^{\frac{1}{\sigma^{2}} \mathbf{x}^{T} \mathbf{s}(\alpha)},
$$

where the signal to noise ratio (SNR) is $1 / \sigma^{2}$.

The Neyman Pearson (NP) detector is a commonly used detector which maximizes the correct detection probability subject to a fixed false alarm probability $[11,12]$. For this detector, a decision rule is obtained by setting a threshold on the (unconditional) likelihood ratio, i.e.,

$$
L(\mathbf{x})<L_{0} \rightarrow K
$$

where $L(\mathbf{x})=\mathrm{E}[\mathrm{L}(\mathbf{x} \mid \alpha)]$. Since the density of $\alpha$ is uniform, the decision rule reduces to an average,

$$
\sum_{\alpha=0}^{Q-1} e^{\frac{1}{\sigma^{2} x^{T} s(\alpha)}<L_{n p} \rightarrow H}
$$

where the constants are incorporated in the threshold.

Another detector that is used commonly is the maximum likelihood (ML) detector $[11,12]$. The decision rule for the ML Detector is

$$
\max _{\alpha}\{L(\mathbf{x} \mid \alpha)\}=\max _{\alpha}\left\{e^{-\frac{1}{2} \mathrm{SNR}} e^{\frac{1}{\sigma^{2}} \mathbf{x}^{T} \mathbf{s}(\alpha)}\right\}<L_{0} \rightarrow H
$$

Since the exponential is a monotonically increasing function, equivalently, the decision rule can be writtcn in terms of the log likelihood ratio,

$$
\max _{\alpha}\left\{\mathrm{x}^{T} \mathrm{~s}(\alpha)\right\}<l_{m l} \rightarrow K
$$

where the constants are incorporated in the threshold.

Next, we consider an image with a chirp-like feature. Assume the test image $s(a, b, q, \theta)$ is a vector of the form,

$$
\begin{gathered}
\mathbf{s}(a, b, q, \theta)=\left[s_{00}(a, b, q, \theta) s_{01}(a, b, q, \theta) \ldots s_{0 M}(a, b, q, \theta)\right. \\
\left.\ldots s_{M 0}(a, b, q, \theta) s_{M 1}(a, b, q, \theta) \ldots s_{M M}(a, b, q, \theta)\right]^{T}
\end{gathered}
$$

obtained by sampling an image function $s$,

$$
\begin{aligned}
s_{m n}(a, b, q, \theta)= & \psi\left(\frac{x_{m} \cos \theta+y_{n} \sin \theta-b}{a}\right) \\
& \times W_{\lambda}\left(-x_{m} \sin \theta+y_{n} \cos \theta-q\right),
\end{aligned}
$$


and normalizing the vector, $\mathbf{s}(a, b, q, \theta)^{T} \mathbf{s}(a, b, q, \theta)=1$. For this problem, we assume the length of chirp-like feature $\lambda$ is known and the location, orientation, and scale given by the coordinates, $a, b, q$, and $\theta$ are unknown. In addition, we assume the random parameters, $a, b, q$, and $\theta$ are independent and contained in the sets

$$
\begin{aligned}
\Omega_{a} & =\left\{a \mid a_{0}, a_{1}, \ldots, a_{S-1}\right\} \\
\Omega_{b} & =\left\{b \mid b_{0}, b_{1}, \ldots, b_{T-1}\right\} \\
\Omega_{q} & =\left\{q \mid q_{0}, \theta_{1}, \ldots, q_{U-1}\right\} \\
\Omega_{\theta} & =\left\{\theta \mid \theta_{0}, \theta_{1}, \ldots, \theta_{V-1}\right\}
\end{aligned}
$$

where each element of the set is assumed to have equal probability.

The detection problem for the sample image is a special case of the detection problem defined earlier where $\alpha=$ $(a, b, q, \theta)$. So,

$$
\Omega_{(a, b, q, \theta)}=\left\{(a, b, q, \theta) \mid a \in \Omega_{a}, b \in \Omega_{b}, q \in \Omega_{q}, \theta \in \Omega_{\theta}\right\} .
$$

For a line segment, the quantity $\mathrm{x}^{T} \mathrm{~s}(a, b, q, \theta)$ can be simplified using the LRTWF,

$$
\mathrm{x}^{T} \mathrm{~s}(a, b, q, \theta)=\sum_{p_{0}=p-w / 2}^{p+w / 2-1}\left(T^{l+t w f} s\right)(a, b, q, \theta)
$$

where $\left(T^{l r t w f} s\right)$ is the LRTWF of the image, as defined in Equation (4). The decision rule for NP Detector in Equation (10) can be written in terms of the LRTWF as

$$
\begin{array}{r}
\sum_{a=0}^{Q-1} \exp \left(-\frac{1}{\sigma^{2}}\left(\sum_{p_{0}=p_{s}-w / 2}^{p_{s}+w / 2-1}\left(T^{i r t w f} s\right)(a, b, q, \theta)\right)\right) \\
<L_{n p} \rightarrow H \\
>K .
\end{array}
$$

Similarly, the decision rule for the ML detector for the sample image can be written in terms of the LRTWF,

$$
\begin{aligned}
& \max _{(a, b, q, \theta)}\left\{\sum_{p_{0}=p-w / 2}^{p+w / 2-1}\left(T^{l \boldsymbol{r} t w f} s\right)(a, b, q, \theta)\right\} \\
& \begin{array}{l}
<l_{m l} \rightarrow H \\
>K .
\end{array}
\end{aligned}
$$

Vext, we consider a chirp-like function shown in Figure 2 for two values of $\lambda$. The results for the NP and ML detector are shown in Figure 3 with

$$
\begin{aligned}
& \Omega_{a}=\{a \mid .25, .5, \ldots, 4\} \\
& \Omega_{b}=\{b \mid-2,-1.875, \ldots, 1.875\} \\
& \Omega_{q}=\{q \mid-2,-1.875, \ldots, 1.875\} \\
& \Omega_{\theta}=\{\theta \mid 0,45, \ldots, 315\}
\end{aligned}
$$

In all three cases, when the SNR is above $13 \mathrm{~dB}$ the performance of the detector is very good.

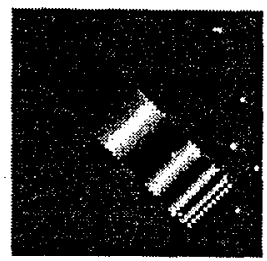

(a)

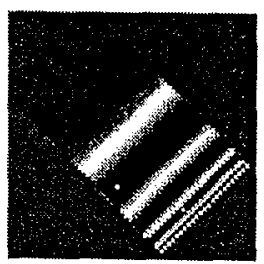

(b)
Figure 2. Sample images with (a) $\lambda=16$ pixels and (b) $\lambda=32$ pixels.

The effect of error on the assumed length of the feature, $\lambda$, for this image is shown in Figure 4. The performance of the detector degrades when an incorrect feature length is assumed. For example, when $\lambda=16$ pixels, the true length of the feature, the performance of the detector is best for both $13 \mathrm{~dB}$ and $16 \mathrm{~dB}$. As the value of $\lambda$ changes, the performance of the detector is not as good. The worst performance occurs when $\lambda$ has the largest amount of error.

\section{SUMMARY}

Internal wakes occur when a ship travels in a stratified media, producing a " $\mathrm{V}$ " shape extending from the ship and a chirp-like feature across each arm. In this paper, a localized Radon transform with a wavelet filter was developed which accentuates both the linear and chirp-like features in images simultaneously. The LRTWF was then incorporated into optimal and sub-optimal detection schemes for images with both linear and chirp-like features contaminated by additive Gaussian noise. Results illustrating the performance of this detector and the effect of error on the length of the linear feature were provided.

\section{REFERENCES}

[1] S. R. Deans, The Radon Transform and Some of Its Applications. New York: John Wiley \& Sons, Inc., 1983.

[2] A. K. Jain, Fundamentals of Digital Image Processing. Englewood Cliffs, NJ: Prentice-Hall, Inc., 1989.

[3] A. C. Copeland, G. Ravichandran, and M. M. Trivedi, "Localized radon transform-based detection of ship wakes in sar images," IEEE Transactions of Geoscience and Remote Sciences, vol. 33, January 1995.

[4] L. M. Murphy, "Linear feature detection and enhancement in noisy images via the radon transform," Pattern Recognition Letters, vol. 4, September 1986.

[5] M. T. Rey, J. K. Tunaley, J. T. Folinsbee, P. A. Jahans, J. A. Dixon, and M. R. Vant, "Application of radon transform techniques to wake detection in seasat-a sar images," IEEE Transactions of Geoscience and Remote Scicnces, vol. 28, July 1990.

[6] J. Hal Beck, Dan Bergondy and Hamed Sari-Sarraf, "Multiresolution segmentation of forward looking ir and sar imagery using neural networks," in Intelligent Robots and Computer Vision IX: Algorithms and Techniques, (Boston, Massacusetts), November 5-7 1991. 


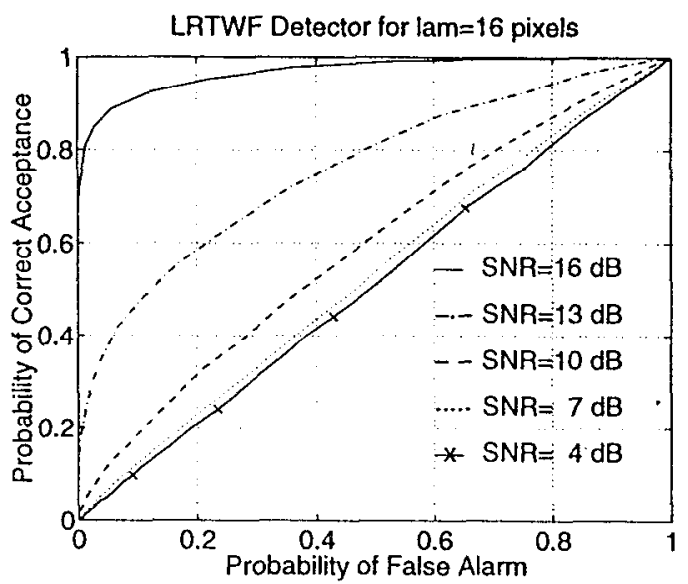

(a)

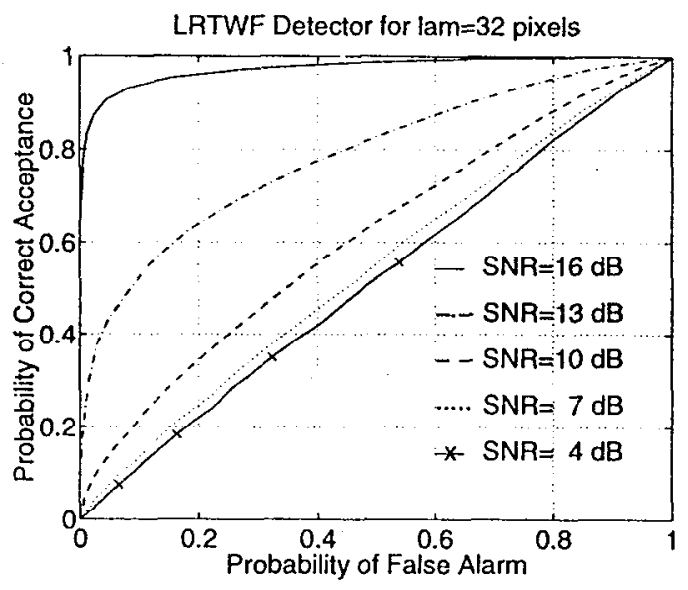

(b)

Figure 3. Performance of the LRTWF detector for the chirp-like function for two line lengths.

[7] Li-jen Du and K. Hoppel, "Multiresolution analysis of sar images using wavelet transform representation," in 1991 International Geoscienc and Remote Sensing Symposium, (Espoo, Finland), June 3-6 1991.

[8] S. K. Rogers, D. W. Ruck, and e. Gregory L. Tarr, "Synthetic aperture radar segmentation using wavelets and fractals," in 1991 IEEE International Conference on Systems Engineering, (Fairborn, Ohio), August 1-3 1991.

[9] J. G. Teti, Jr. and H. N. Kritikos, "Sar ocean image representation using wavelets," IEEE Transaction on Geoscience and Remote Sensing, vol. 30, September 1992.

[10] I. Daubechies, Ten Lectures on Wavelets. Philadelphia, PA: Society for Industrial and Applied Mathematics, 1992.

[11] H. L. Van Trees, Detection, Estimation, and Modulation Theory. New York: John Wiley \& Sons, Inc., 1971.

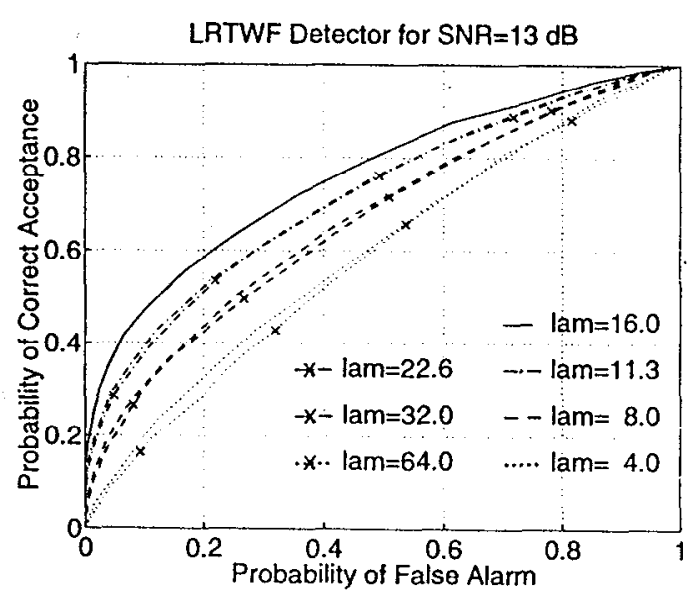

(a)

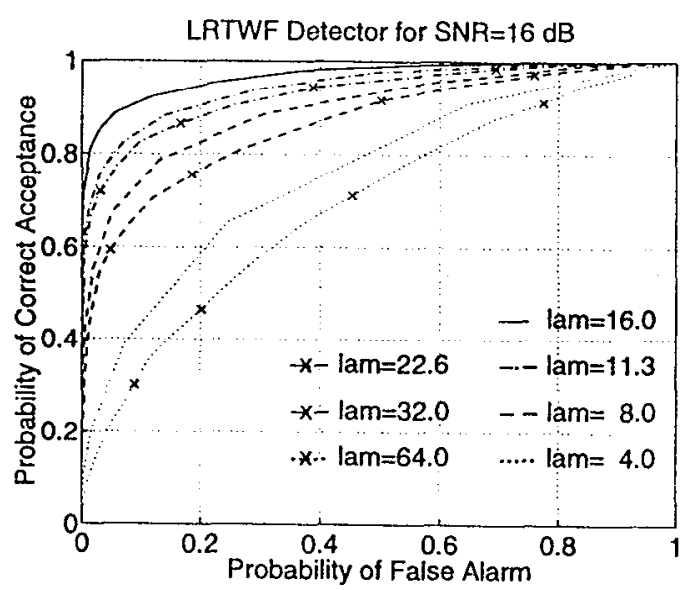

(b)

Figure 4. Effect of error on $\lambda$ on the performance of the LRTWF detector for the chirp-like function with a line length $\lambda=16$ pixels when (a) $S N R=13 \mathrm{~dB}$ and (b) $\mathrm{SNR}=16 \mathrm{~dB}$.

[12] H. V. Poor, An Introduction to Signal Detection and Estimation. New York: Dowden \& Culver, Inc., 1994.

\section{Acknowledgments}

This work was supported in part by Lawrence Livermore National Laboratory Contract B277495. 